\title{
Correction to: Increasing water productivity, nitrogen economy, and grain yield of rice by water saving irrigation and fertilizer-N management
}

\author{
Omar Aziz ${ }^{1}$. Saddam Hussain ${ }^{2} \cdot$ Muhammad Rizwan $^{3} \cdot$ Muhammad Riaz $^{1} \cdot$ Saqib Bashir $^{1} \cdot$ Lirong Lin $^{1}$. \\ Sajid Mehmood ${ }^{1} \cdot$ Muhammad Imran $^{1} \cdot$ Rizwan Yaseen $^{4} \cdot$ Guoan Lu ${ }^{1}$
}

Published online: 1 May 2018

(C) Springer-Verlag GmbH Germany, part of Springer Nature 2018

\section{Correction to: Environ Sci Pollut Res \\ https://doi.org/10.1007/s11356-018-1855-z}

The original publication of this paper contains a mistake.

Correct presentation of Tables 4, 5 and 6 is shown in this article.

The original article has been corrected.

The online version of the original article can be found at https://doi.org/ $10.1007 / \mathrm{s} 11356-018-1855-\mathrm{z}$

Guoan $\mathrm{Lu}$

glu@mail.hzau.edu.cn

1 College of Resources and Environment, Huazhong Agricultural University, Wuhan 430070, China

2 Department of Agronomy, University of Agriculture Faisalabad, Faisalabad 38040, Pakistan

3 Microelement Research Center, Huazhong Agricultural University, Wuhan 430070, China

4 Department of Soil Science, Faculty of Agricultural Sciences and Technology, Bahauddin Zakariya University, Multan 60800, Pakistan 
Table 4 Dry biomass of different parts of rice and grain yield in conventional irrigation (CI) and "thin-shallow-moist-dry" irrigation (TSMDI) and different $\mathrm{N}$ inputs at successive characteristic stages i.e. (a) tillering, (b) booting, and (c) post-harvest

\begin{tabular}{|c|c|c|c|c|c|c|}
\hline \multicolumn{2}{|l|}{ Treatment } & \multirow{2}{*}{$\begin{array}{l}\text { Root dry biomass } \\
\left.\text { (g plant }^{-1}\right)\end{array}$} & \multirow{2}{*}{$\begin{array}{l}\text { Straw dry biomass } \\
\left(\text { g plant }^{-1}\right)\end{array}$} & \multirow{2}{*}{$\begin{array}{l}\text { Leaf dry biomass } \\
\left(\text { g plant }^{-1}\right)\end{array}$} & \multirow{2}{*}{$\begin{array}{l}\text { Grain yield } \\
\left(\mathrm{g} \text { plant }^{-1}\right)\end{array}$} & \multirow{2}{*}{$\begin{array}{l}\text { Total biomas } \\
\left(\mathrm{g} \mathrm{plant}^{-1}\right)\end{array}$} \\
\hline Irrigation management & $\mathrm{N}$ inputs & & & & & \\
\hline \multicolumn{7}{|l|}{$\mathrm{a}$} \\
\hline \multirow[t]{3}{*}{$\mathrm{CI}$} & $\mathrm{N}_{0}$ & $1.586 \mathrm{~d}$ & $4.150 \mathrm{c}$ & $1.910 \mathrm{c}$ & & $7.647 \mathrm{c}$ \\
\hline & $\mathrm{N}_{1}$ & $1.993 \mathrm{c}$ & $7.310 \mathrm{~b}$ & $3.433 b$ & & $12.740 \mathrm{~b}$ \\
\hline & $\mathrm{N}_{2}$ & $2.346 \mathrm{~b}$ & $9.446 \mathrm{a}$ & $4.292 \mathrm{a}$ & & $16.087 \mathrm{a}$ \\
\hline \multirow[t]{3}{*}{ TSMDI } & $\mathrm{N}_{0}$ & $1.640 \mathrm{~d}$ & $4.123 \mathrm{c}$ & $1.881 \mathrm{c}$ & & $7.643 \mathrm{c}$ \\
\hline & $\mathrm{N}_{1}$ & $2.056 \mathrm{c}$ & $7.663 b$ & $3.441 \mathrm{~b}$ & & $13.160 b$ \\
\hline & $\mathrm{N}_{2}$ & $2.436 \mathrm{a}$ & $9.170 \mathrm{a}$ & $4.244 \mathrm{a}$ & & $15.853 \mathrm{a}$ \\
\hline \multicolumn{7}{|l|}{ Significance } \\
\hline Irrigation management (I) & & $* *$ & ns & ns & & ns \\
\hline $\mathrm{N}$ inputs $(\mathrm{N})$ & & $* *$ & $* *$ & $* *$ & & $* *$ \\
\hline $\mathrm{I} \times \mathrm{N}$ interaction & & ns & ns & ns & & ns \\
\hline \multicolumn{7}{|l|}{$\mathrm{b}$} \\
\hline \multirow[t]{3}{*}{ CI } & $\mathrm{N}_{0}$ & $2.513 \mathrm{e}$ & $12.123 \mathrm{c}$ & $3.076 \mathrm{c}$ & & $17.713 \mathrm{c}$ \\
\hline & $\mathrm{N}_{1}$ & $3.570 \mathrm{~d}$ & $15.780 \mathrm{~b}$ & $4.083 b$ & & $23.433 b$ \\
\hline & $\mathrm{N}_{2}$ & $5.093 \mathrm{~b}$ & $19.667 \mathrm{a}$ & $5.196 \mathrm{a}$ & & $29.957 \mathrm{a}$ \\
\hline \multirow[t]{3}{*}{ TSMDI } & $\mathrm{N}_{0}$ & $2.620 \mathrm{e}$ & $11.110 \mathrm{c}$ & $2.920 \mathrm{c}$ & & $16.650 \mathrm{c}$ \\
\hline & $\mathrm{N}_{1}$ & $3.940 \mathrm{c}$ & $14.913 b$ & $3.953 b$ & & $22.807 b$ \\
\hline & $\mathrm{N}_{2}$ & $5.860 \mathrm{a}$ & $18.473 \mathrm{a}$ & $4.930 \mathrm{a}$ & & $29.263 \mathrm{a}$ \\
\hline \multicolumn{7}{|l|}{ Significance } \\
\hline Irrigation management (I) & & $* *$ & $*$ & ns & & ns \\
\hline $\mathrm{N}$ inputs $(\mathrm{N})$ & & $* *$ & $* *$ & $* *$ & & $* *$ \\
\hline $\mathrm{I} \times \mathrm{N}$ interaction & & ns & ns & ns & & ns \\
\hline \multicolumn{7}{|l|}{$\mathrm{c}$} \\
\hline \multirow[t]{3}{*}{$\mathrm{CI}$} & $\mathrm{N}_{0}$ & $1.286 \mathrm{c}$ & $8.450 \mathrm{e}$ & $2.283 \mathrm{~d}$ & $19.080 \mathrm{c}$ & $31.100 \mathrm{c}$ \\
\hline & $\mathrm{N}_{1}$ & $2.066 \mathrm{~b}$ & $13.297 \mathrm{c}$ & $3.926 \mathrm{bc}$ & $28.614 b$ & $47.903 b$ \\
\hline & $\mathrm{N}_{2}$ & $3.383 \mathrm{a}$ & $16.833 \mathrm{a}$ & $4.896 \mathrm{a}$ & $40.190 \mathrm{a}$ & $65.303 \mathrm{a}$ \\
\hline \multirow[t]{3}{*}{ TSMDI } & $\mathrm{N}_{0}$ & $1.440 \mathrm{c}$ & $7.470 \mathrm{f}$ & $2.240 \mathrm{~d}$ & $19.753 c$ & $30.903 c$ \\
\hline & $\mathrm{N}_{1}$ & $2.416 b$ & $12.107 d$ & $3.166 \mathrm{c}$ & $29.247 b$ & $46.937 b$ \\
\hline & $\mathrm{N}_{2}$ & $3.753 \mathrm{a}$ & $15.690 \mathrm{~b}$ & $4.343 \mathrm{ab}$ & $40.883 a$ & $64.670 \mathrm{a}$ \\
\hline \multicolumn{7}{|l|}{ Significance } \\
\hline Irrigation management (I) & & $*$ & $* *$ & * & ns & ns \\
\hline $\mathrm{N}$ inputs $(\mathrm{N})$ & & $* *$ & $* *$ & $* *$ & $* *$ & $* *$ \\
\hline $\mathrm{I} \times \mathrm{N}$ interaction & & ns & ns & ns & ns & ns \\
\hline
\end{tabular}

Means followed by the same letter in each column are not significantly different at $P<0.05$ (LSD test). $\mathrm{N}_{0}=0 \mathrm{~kg} \mathrm{~N} \mathrm{ha}^{-1}, \mathrm{~N}_{1}=90 \mathrm{~kg} \mathrm{~N}^{-1}, \mathrm{~N}_{2}=$ $180 \mathrm{~kg} \mathrm{~N} \mathrm{ha}^{-1}$

ns not significant at $P>0.05$

* Significant at $P<0.05$

** Significant at $P<0.01$ 
Table 5 Nitrogen uptake by different parts of rice in conventional irrigation (CI) and "thin-shallow-moist-dry" irrigation (TSMDI) and different N inputs at successive characteristic stages i.e. (a) tillering, (b) booting, and (c) post-harvest

\begin{tabular}{|c|c|c|c|c|c|c|}
\hline \multicolumn{2}{|l|}{ Treatment } & \multirow{2}{*}{$\begin{array}{l}\text { Root } \mathrm{N} \text { uptake } \\
\left(\mathrm{g} \text { plant }^{-1}\right)\end{array}$} & \multirow{2}{*}{$\begin{array}{l}\text { Straw N uptake } \\
\left(\text { g plant }^{-1}\right)\end{array}$} & \multirow{2}{*}{$\begin{array}{l}\text { Leaf N uptake } \\
\left(\mathrm{g} \text { plant }^{-1}\right)\end{array}$} & \multirow{2}{*}{$\begin{array}{l}\text { Grain N uptake } \\
\left(\text { g plant }^{-1}\right)\end{array}$} & \multirow{2}{*}{$\begin{array}{l}\text { Total N uptake } \\
\left(\text { g plant }^{-1}\right)\end{array}$} \\
\hline Irrigation management & $\mathrm{N}$ inputs & & & & & \\
\hline \multicolumn{7}{|l|}{$\mathrm{a}$} \\
\hline \multirow[t]{3}{*}{ CI } & $\mathrm{N}_{0}$ & $0.0115 \mathrm{~d}$ & $0.0275 \mathrm{c}$ & $0.0364 \mathrm{c}$ & & $0.0754 \mathrm{c}$ \\
\hline & $\mathrm{N}_{1}$ & $0.0166 \mathrm{c}$ & $0.0556 \mathrm{~b}$ & $0.0725 b$ & & $0.1448 b$ \\
\hline & $\mathrm{N}_{2}$ & $0.0224 \mathrm{~b}$ & $0.0866 \mathrm{a}$ & $0.1087 \mathrm{a}$ & & $0.2177 \mathrm{a}$ \\
\hline \multirow[t]{3}{*}{ TSMDI } & $\mathrm{N}_{0}$ & $0.0119 \mathrm{~d}$ & $0.0276 \mathrm{c}$ & $0.0373 \mathrm{c}$ & & $0.0769 \mathrm{c}$ \\
\hline & $\mathrm{N}_{1}$ & $0.0175 \mathrm{c}$ & $0.0600 \mathrm{~b}$ & $0.0742 b$ & & $0.1517 \mathrm{~b}$ \\
\hline & $\mathrm{N}_{2}$ & $0.0241 \mathrm{a}$ & $0.0820 \mathrm{a}$ & $0.1080 \mathrm{a}$ & & $0.2140 \mathrm{a}$ \\
\hline \multicolumn{7}{|l|}{ Significance } \\
\hline Irrigation management (I) & & $*$ & ns & ns & & ns \\
\hline $\mathrm{N}$ inputs $(\mathrm{N})$ & & $* *$ & $* *$ & $* *$ & & $* *$ \\
\hline $\mathrm{I} \times \mathrm{N}$ interaction & & ns & ns & ns & & ns \\
\hline \multicolumn{7}{|l|}{$\mathrm{b}$} \\
\hline \multirow[t]{3}{*}{ CI } & $\mathrm{N}_{0}$ & $0.0135 \mathrm{e}$ & $0.0735 \mathrm{c}$ & $0.0494 \mathrm{c}$ & & $0.1365 \mathrm{c}$ \\
\hline & $\mathrm{N}_{1}$ & $0.0223 \mathrm{~d}$ & $0.1136 b$ & $0.0688 \mathrm{~b}$ & & $0.2046 b$ \\
\hline & $\mathrm{N}_{2}$ & $0.0361 \mathrm{~b}$ & $0.1652 \mathrm{a}$ & $0.0991 \mathrm{a}$ & & $0.3004 \mathrm{a}$ \\
\hline \multirow[t]{3}{*}{ TSMDI } & $\mathrm{N}_{0}$ & $0.0149 \mathrm{e}$ & $0.0685 \mathrm{c}$ & $0.0480 \mathrm{c}$ & & $0.1313 \mathrm{c}$ \\
\hline & $\mathrm{N}_{1}$ & $0.0258 \mathrm{c}$ & $0.1089 b$ & $0.0692 b$ & & $0.2039 b$ \\
\hline & $\mathrm{N}_{2}$ & $0.0426 \mathrm{a}$ & $0.1589 \mathrm{a}$ & $0.0976 \mathrm{a}$ & & $0.2991 \mathrm{a}$ \\
\hline \multicolumn{7}{|l|}{ Significance } \\
\hline Irrigation management (I) & & $* *$ & ns & ns & & ns \\
\hline $\mathrm{N}$ inputs $(\mathrm{N})$ & & $* *$ & $* *$ & $* *$ & & $* *$ \\
\hline $\mathrm{I} \times \mathrm{N}$ interaction & & ns & ns & ns & & ns \\
\hline \multicolumn{7}{|l|}{$\mathrm{c}$} \\
\hline \multirow[t]{3}{*}{ CI } & $\mathrm{N}_{0}$ & $0.0065 \mathrm{c}$ & $0.0346 \mathrm{e}$ & $0.0130 \mathrm{c}$ & $0.1744 c$ & $0.2288 \mathrm{c}$ \\
\hline & $\mathrm{N}_{1}$ & $0.0118 b$ & $0.0680 \mathrm{c}$ & $0.0251 \mathrm{~b}$ & $0.2966 b$ & $0.4015 b$ \\
\hline & $\mathrm{N}_{2}$ & $0.0205 \mathrm{a}$ & $0.1091 \mathrm{a}$ & $0.0367 \mathrm{a}$ & $0.4705 \mathrm{a}$ & $0.6369 a$ \\
\hline \multirow[t]{3}{*}{ TSMDI } & $\mathrm{N}_{0}$ & $0.0075 \mathrm{c}$ & $0.0313 \mathrm{f}$ & $0.0133 \mathrm{c}$ & $0.1871 \mathrm{c}$ & $0.2391 \mathrm{c}$ \\
\hline & $\mathrm{N}_{1}$ & $0.0143 b$ & $0.0633 d$ & $0.0206 \mathrm{~b}$ & $0.3140 \mathrm{~b}$ & $0.4121 b$ \\
\hline & $\mathrm{N}_{2}$ & $0.0229 \mathrm{a}$ & $0.1022 b$ & $0.0335 \mathrm{a}$ & $0.4918 \mathrm{a}$ & $0.6504 \mathrm{a}$ \\
\hline \multicolumn{7}{|l|}{ Significance } \\
\hline Irrigation management (I) & & $*$ & $* *$ & ns & ns & ns \\
\hline $\mathrm{N}$ inputs $(\mathrm{N})$ & & $* *$ & $* *$ & $* *$ & $* *$ & $* *$ \\
\hline $\mathrm{I} \times \mathrm{N}$ interaction & & ns & ns & $\mathrm{ns}$ & ns & ns \\
\hline
\end{tabular}

Means followed by the same letter in each column are not significantly different at $P<0.05$ (LSD test). $\mathrm{N}_{0}=0 \mathrm{~kg} \mathrm{~N} \mathrm{ha}^{-1}, \mathrm{~N}_{1}=90 \mathrm{~kg} \mathrm{~N}$ ha ${ }^{-1}, \mathrm{~N}_{2}=$ $180 \mathrm{~kg} \mathrm{~N} \mathrm{ha}^{-1}$

$n s$ not significant at $P>0.05$

* Significant at $P<0.05$

**Significant at $P<0.01$ 
Table 6 Nitrogen derived from fertilizer (Ndff $(\%)$ ) by different parts of rice in conventional irrigation $(\mathrm{CI})$ and "thinshallowmoist- dry" irrigation (TSMDI) and different $\mathrm{N}$ inputs at successive characteristic stages i.e. (a) tillering, (b) booting, and (c) postharvest
Treatment

Root Ndff (\%) Straw Ndff (\%) Leaf Ndff (\%) Grain Ndff $(\%)$

Irrigation management

$\mathrm{N}$ inputs

\section{a}

CI

TSMDI

Significance

Irrigation management (I)

$\mathrm{N}$ inputs $(\mathrm{N})$

$\mathrm{I} \times \mathrm{N}$ interaction

b

$\mathrm{CI}$

TSMDI

Significance

Irrigation management (I)

$\mathrm{N}$ inputs $(\mathrm{N})$

$\mathrm{I} \times \mathrm{N}$ interaction

CI

TSMDI

Significance

Irrigation management (I)

$\mathrm{N}$ inputs $(\mathrm{N})$

$\mathrm{I} \times \mathrm{N}$ interaction

$\begin{array}{llll}\mathrm{N}_{1} & 28.55 \mathrm{a} & 37.25 \mathrm{~b} & 41.16 \mathrm{~b} \\ \mathrm{~N}_{2} & 32.16 \mathrm{ab} & 43.80 \mathrm{a} & 55.11 \mathrm{a} \\ \mathrm{N}_{1} & 29.52 \mathrm{ab} & 38.43 \mathrm{~b} & 43.15 \mathrm{~b} \\ \mathrm{~N}_{2} & 33.62 \mathrm{a} & 42.66 \mathrm{a} & 54.83 \mathrm{a}\end{array}$

$41.16 \mathrm{~b}$

$43.15 b$

$4.83 a$

ns ns ns

* $\quad * *$

ns

ns

**

ns

$\begin{array}{llll}\mathrm{N}_{1} & 24.50 \mathrm{~b} & 30.06 \mathrm{a} & 34.09 \mathrm{~b} \\ \mathrm{~N}_{2} & 28.68 \mathrm{a} & 36.51 \mathrm{a} & 38.91 \mathrm{ab} \\ \mathrm{N}_{1} & 26.03 \mathrm{~b} & 32.25 \mathrm{a} & 35.10 \mathrm{~b} \\ \mathrm{~N}_{2} & 30.60 \mathrm{a} & 38.63 \mathrm{a} & 42.02 \mathrm{a}\end{array}$

$\begin{array}{llll}\mathrm{N}_{2} & 30.60 \mathrm{a} & 38.63 \mathrm{a} & 42.02 \mathrm{a}\end{array}$

* ns ns

** $\quad$ ns $\quad *$

ns ns ns

$\begin{array}{lllll}\mathrm{N}_{1} & 16.97 \mathrm{~b} & 23.56 \mathrm{c} & 25.71 \mathrm{~b} & 27.89 \mathrm{c}\end{array}$

$\begin{array}{lllll}\mathrm{N}_{2} & 20.19 \mathrm{a} & 28.41 \mathrm{~b} & 32.44 \mathrm{a} & 33.70 \mathrm{~b}\end{array}$

$\begin{array}{lllll}\mathrm{N}_{1} & 17.40 \mathrm{~b} & 25.01 \mathrm{c} & 27.93 \mathrm{~b} & 31.48 \mathrm{~b}\end{array}$

$\begin{array}{lllll}\mathrm{N}_{2} & 20.49 \mathrm{a} & 32.67 \mathrm{a} & 34.56 \mathrm{a} & 36.57 \mathrm{a}\end{array}$

Means followed by the same letter in each column are not significantly different at $P<0.05$ (LSD test). $\mathrm{N}_{1}=90 \mathrm{~kg}$

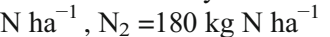

$n s$ not significant at $P>0.05$

* Significant at $P<0.05$

**Significant at $P<0.01$ ns ns

ns

$* *$

ns $\quad * *$

** $\quad * *$

ns ns

$\begin{array}{lll} & \mathrm{ns} & \\ * * & * * & * * \\ \mathrm{~ns} & \mathrm{~ns} & \mathrm{~ns}\end{array}$

